\title{
Performance Analysis of Network-RTK Techniques for Drone Navigation considering Ionospheric Conditions
}

\author{
Tae-Suk Bae $(\mathbb{D})$ and Minho Kim \\ Department Geoinformation Engineering, Sejong University, Seoul 05006, Republic of Korea \\ Correspondence should be addressed to Tae-Suk Bae; baezae@sejong.ac.kr
}

Received 26 February 2018; Accepted 15 April 2018; Published 10 May 2018

Academic Editor: Hyung-Sup Jung

Copyright (c) 2018 Tae-Suk Bae and Minho Kim. This is an open access article distributed under the Creative Commons Attribution License, which permits unrestricted use, distribution, and reproduction in any medium, provided the original work is properly cited.

\begin{abstract}
Recently, an accurate positioning has become the kernel of autonomous navigation with the rapid growth of drones including mapping purpose. The Network-based Real-time Kinematic (NRTK) system was predominantly used for precision positioning in many fields such as surveying and agriculture, mostly in static mode or low-speed operation. The NRTK positioning, in general, shows much better performance with the fixed integer ambiguities. However, the success rate of the ambiguity resolution is highly dependent on the ionospheric condition and the surrounding environment of Global Navigation Satellite System (GNSS) positioning, which particularly corresponds to the low-cost GNSS receivers. We analyzed the effects of the ionospheric conditions on the GNSS NRTK, as well as the possibility of applying the mobile NRTK to drone navigation for mapping. Two NRTK systems in operation were analyzed during a period of high ionospheric conditions, and the accuracy and the performance were compared for several operational cases. The test results show that a submeter accuracy is available even with float ambiguity under a favorable condition (i.e., visibility of the satellites as well as stable ionosphere). We still need to consider how to deal with ionospheric disturbances which may prevent NRTK positioning.
\end{abstract}

\section{Introduction}

The Global Navigation Satellite System (GNSS) is widely used for accurate positioning in various civil applications. The stand-alone positioning using C/A code pseudoranges is the most popular approach for aviation and/or ground vehicle navigation. However, a submeter or better accuracy is required for Mobile Mapping System (MMS) or smart cars for identifying lanes, especially, the Lane Departure Warning System (LDWS) or Advanced Driver Assistance Systems (ADAS). Contrary to the ground vehicle, there is no designated paths for drones, which make it difficult to provide accurate control points. Rather than code-based GNSS positioning in either stand-alone or differential mode, the carrier phase observables are commonly used for precision geodetic and surveying applications where an accurate positioning is the most important consideration. A Realtime Kinematic (RTK) approach can provide a centimeter- level accuracy in a favorable environment. A more accurate and stable positioning technique was first proposed in mid-1990 by rigorously modeling all error components based on a network of known references, called Networkbased RTK (NRTK) technique [1-3]. Since then, several methods were developed and applied in practice such as Virtual Reference Station (VRS), Flächen Korrektur Parameter (FKP), and Master-Auxiliary Concept (MAC), which are basically similar approaches [4-7].

With a widespread use of drones in the field of geoinformation, we need to understand the accuracy requirements for the topographic mapping purpose in an open sky. For an accurate position of the camera exposure, it is necessary to achieve decimeter accuracy of the drones in 3-dimensional. Even with the carrier phase observations, it is challenging to reach such an accuracy without proper integer ambiguity resolution. However, it is well known that fixing integer ambiguity of the carrier phase 
signals is difficult, or sometimes exaggerated errors may occur on the resolved ambiguities depending on the geometric configuration and/or surrounding environment.

Therefore, we analyzed the stability and the accuracy of NRTK systems under various conditions to verify the possibility of applying this to drone navigation. We used the currently available NRTK systems in Korea for the test. The National Geographic Information Institute (NGII) of Korea is operating two NRTK services, that is, VRS and FKP $[3,8]$. The reference stations of Korea are densely located ranging from 30 to $50 \mathrm{~km}$ for most of the baselines, which should be a worthy infrastructure for NRTK positioning.

\section{NRTK System}

2.1. Overview of NRTK System. The accuracy and stability of NRTK are considerably affected by the ambiguity resolution of the carrier phases [9-11]. However, it is difficult to accomplish accurate resolution due to many factors such as insufficient number of observations, the distance to the reference stations, the signal blockage near the moving rover, and the erratic ionospheric condition [12]. Therefore, it is necessary to understand the relationship with an external information (e.g., ionospheric conditions) to apply for drone navigation, especially for topographic mapping purposes in an open sky. These analyses were conducted in two ways, that is, (1) internal factors of NRTK technique and GNSS receiver specification and (2) the satellite configuration, the behavior of the rover, and the space radio signal disturbance as external factors.

The NRTK correction information is generally transmitted to the user (mostly the rover) via Radio Technical Commission for Maritime Services (RTCM) format, although the proprietary format such as Compact Measurement Record (CMR) or CMR+ is also available [12-14]. The RTCM Special Committee (SC) 104 on Differential GNSS (DGNSS) provides the standards for differential GPS and RTK operations. The RTCM was mainly used for the correction information in this study.

2.2. NRTK Reference Network of Korea. The actual NRTK surveying was conducted for kinematic positioning of ground vehicles across the country, instead of a real drone like a multicopter, due to the limitation of payload of the receivers. In addition, the static NRTK positioning was also carried out using zero-baseline configuration. The reference network of Korea at the time of the experiment is shown in Figure 1.

Although two NRTK systems were operational by the same institute (NGII), the reference network for the two systems is not the same due to the different licensing policy. In addition, the spatial density of the NRTK reference network is not identical to both systems. While VRS uses all Continuously Operating Reference Stations (CORS) for modeling of the correction information, only 30 CORS are involved in the network of FKP. The performance and the stability of the two systems can change depending on the internal and/or external environment of the receiving system (mostly the rover). The status of the rover can be either static

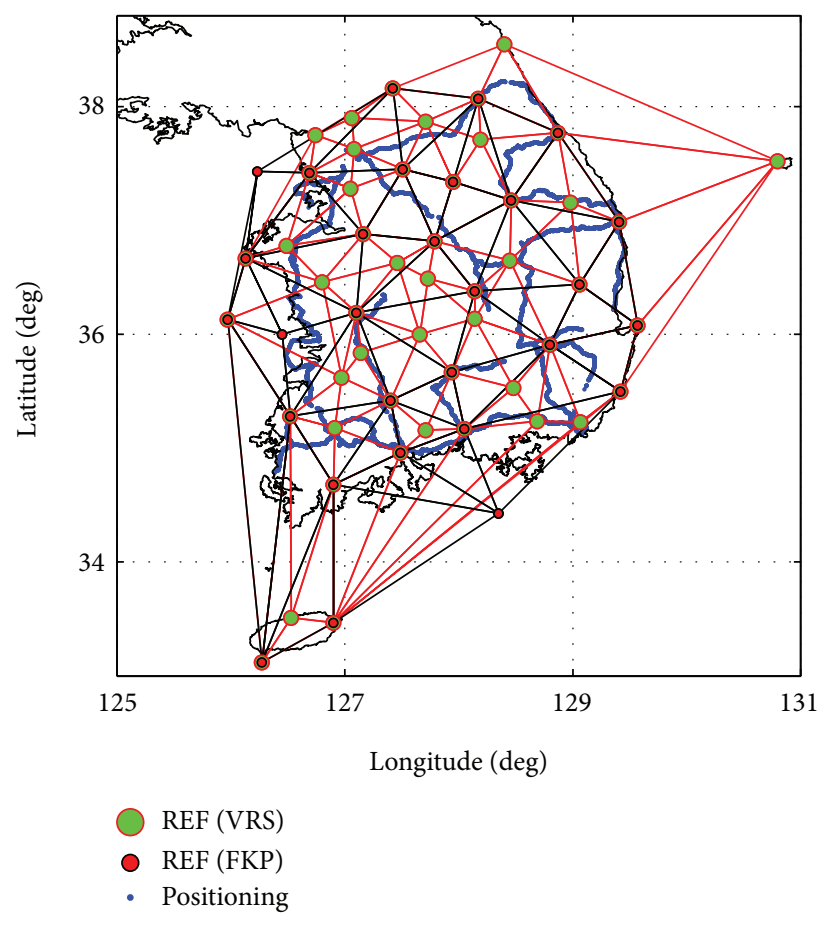

FIGURE 1: Configuration of NRTK network of Korea [8].

(stationary) or kinematic (moving), and the same condition should be guaranteed during the experiment except the NRTK techniques.

2.3. System Configuration of the Study. We set up a system to test NRTK performance and stability in three cases. Figure 2 shows the basic system configuration where the GNSS antenna (Leica GRX1200) was installed in an open sky rooftop by splitting the signal to ensure the identical condition of the two systems. The correction information was sent to the receiver via serial port, and the NRTK solutions were transferred back to the main computer through the same ports. Each receiver was set up to conduct NRTK positioning with the corresponding correction information of VRS or FKP.

The two other cases of the study have basically the same system configuration, but some modification was applied to the GNSS receivers, antenna, and/or the mode of operation (static or kinematic).

\section{Experiments}

It is generally known that the integer ambiguity resolution is essential for high-accuracy NRTK solutions based on the carrier phase observations. We used a dual-frequency GNSS receiver as well as a single-frequency low-cost receiver for static and/or kinematic positioning. Three experiments were conducted to evaluate the performance of NRTK systems in different environments and the effect of positioning modes (see Table 1). First, since the NRTK performance is generally affected by the atmosphere $[15,16]$, especially ionospheric disturbances, we analyzed two types of NRTK systems under restricted experimental conditions (case 1). For this 


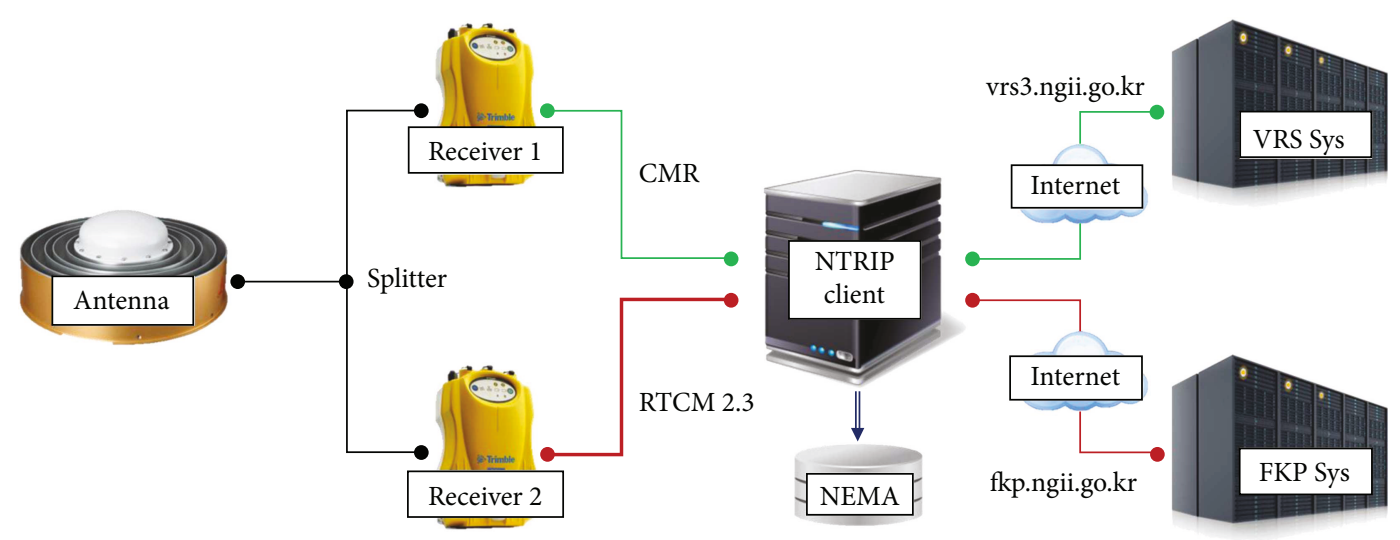

FIGURE 2: Systematic configuration for the comparison of static NRTK solutions (VRS versus FKP) [3].

TABLE 1: Overview of the experiment for NRTK positioning.

\begin{tabular}{|c|c|c|c|c|c|c|}
\hline \multirow[b]{2}{*}{ Component } & \multicolumn{2}{|c|}{ Case 1} & \multicolumn{2}{|c|}{ Case 2} & \multicolumn{2}{|c|}{ Case 3} \\
\hline & VRS & FKP & VRS & FKP & VRS & VRS \\
\hline Receiver & \multicolumn{2}{|c|}{ Trimble 5700} & \multicolumn{2}{|c|}{ Trimble R6 } & Trimble R6 & ublox EVK-6T \\
\hline Frequency & \multicolumn{2}{|c|}{$\mathrm{L} 1 / \mathrm{L} 2$} & \multicolumn{2}{|c|}{$\mathrm{L} 1 / \mathrm{L} 2$} & $\mathrm{~L} 1 / \mathrm{L} 2$ & L1 \\
\hline Sampling & \multicolumn{2}{|c|}{$1 \mathrm{~Hz}$} & \multicolumn{2}{|c|}{$1 \mathrm{~Hz}$} & \multicolumn{2}{|c|}{$1 \mathrm{~Hz}$} \\
\hline Period & \multicolumn{2}{|c|}{1 month } & \multicolumn{2}{|c|}{5 days } & \multicolumn{2}{|c|}{$\begin{array}{c}1.5 \mathrm{~h} \text { (ublox) } \\
11 \text { days (Trimble R6) }\end{array}$} \\
\hline GNSS & \multicolumn{2}{|c|}{ GPS } & \multicolumn{2}{|c|}{ GPS + GLO } & GPS + GLO & GPS \\
\hline Antenna & \multicolumn{2}{|c|}{ Leica AT504GG } & \multicolumn{2}{|c|}{ Internal } & Internal & Patch type \\
\hline Protocol & CMR & RTCM 3.1 & CMR & RTCM 3.1 & & 3.1 \\
\hline Common epochs & \multicolumn{2}{|c|}{165,719} & \multicolumn{2}{|c|}{7516} & \multicolumn{2}{|c|}{3462} \\
\hline Fixed epochs & \multicolumn{2}{|c|}{116,023} & \multicolumn{2}{|c|}{6989} & 3251 & 306 \\
\hline Software & Internal & Internal & Internal & Internal & Internal & RTKLIB \\
\hline
\end{tabular}

experiment, the same type of GNSS receivers was connected to a single antenna on the rooftop of a building by splitting the signals. The main objective is to evaluate the effect of ionospheric conditions on the NRTK performance.

The second test (case 2) was carried out in a static mode where both receivers were connected to a single antenna, similarly to case 1 . In this case, however, we analyzed the performance of two NRTK techniques with identical receivers, as well as different grades of GNSS receivers. The last experiment (case 3 ) is a kinematic NRTK positioning with a low-cost receiver with respect to a geodetic grade receiver. Every single epoch of NRTK solutions was analyzed for 24 hours. In kinematic positioning, the success rate of the ambiguity resolution was mainly evaluated along with relative baselines with respect to the reference solution. All experiments used the RTCM message as a principal data format, and CMR/CMR+ was also used for some VRS operations.

3.1. Effect of Ionospheric Condition (Case 1). As already known in GNSS community, the double-differenced observables can eliminate the satellite and receiver clock biases, orbit errors, and interchannel biases [17]. However, atmospheric delays, such as ionosphere and troposphere, still remain in the observables; thus, these should be eliminated for precision positioning and stability of the system. A new approach was proposed to deal with these atmospheric delays in RTK [1]. It is difficult to eliminate the ionospheric residual errors even with NRTK when the atmospheric disturbance occurs due to a solar flare. Therefore, it is still a critical issue to minimize the effects of space radio signal disturbances on NRTK. The Korean Space Weather Center (KSWC) forecasts the disturbance grades for every event. As can be seen in Table 2, several geomagnetic disturbances and radio blackouts happened during the entire period of the experiment from September 26 to October 31, 2013. We compared the effects of solar flares, radio signal blockages, and geomagnetic disturbance on NRTK and analyzed the degradation of the system performance by these disturbances.

There was no significant degradation of the NRTK accuracy and stability in the case of the disturbance stage 1 (see Figure 3). However, the FKP system seems not to work properly for stage 2 or 3. Particularly, two warnings in stage 3 on October 25, 2013, prohibited the users from accessing the FKP server, and there were cases that the performance was highly correlated with the disturbances. Therefore, it is necessary to consider the signal disturbances to apply NRTK for the navigation of the drones. 
TABLE 2: Disturbances and radio signal interruptions during the experiment [8].

\begin{tabular}{|c|c|c|c|c|}
\hline Type & Local time (start) & Grade & Local time (end) & Max. value \\
\hline Geomagnetic disturbances & 2013-10-09 06:00 & G1 & 2013-10-09 15:00 & $\mathrm{Kp}=5$ \\
\hline Geomagnetic disturbances & 2013-10-09 18:00 & G1 & 2013-10-09 21:00 & $\mathrm{Kp}=5$ \\
\hline Radio blackout & 2013-10-24 09:26 & $\mathrm{R} 2$ & 2013-10-24 09:36 & M9.3 \\
\hline Radio blackout & 2013-10-25 16:59 & R3 & 2013-10-25 17:09 & $\mathrm{X} 1.7$ \\
\hline Radio blackout & $2013-10-2523: 58$ & R3 & 2013-10-26 00:12 & $\mathrm{X} 2.1$ \\
\hline Radio blackout & 2013-10-28 10:58 & $\mathrm{R} 2$ & - & M9.5 \\
\hline Radio blackout & 2013-10-28 11:02 & R3 & 2013-10-28 11:13 & $\mathrm{X} 1.0$ \\
\hline Radio blackout & 2013-10-28 13:41 & $\mathrm{R} 2$ & $2013-10-28$ 13:46 & M5.1 \\
\hline Radio blackout & 2013-10-30 06:48 & $\mathrm{R} 2$ & - & M9.4 \\
\hline Radio blackout & $2013-10-3006: 50$ & R3 & 2013-10-30 07:01 & $\mathrm{X} 2.3$ \\
\hline
\end{tabular}

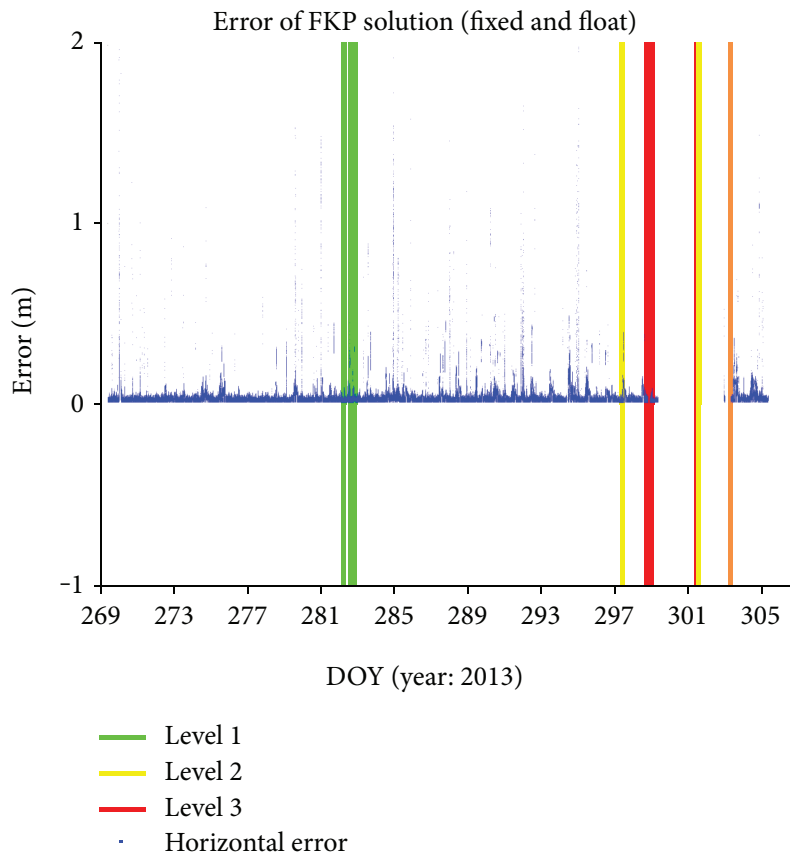

FIGURE 3: NRTK positioning errors (fixed and float solutions) with respect to the ionospheric disturbances [3].

3.2. Characteristics of NRTK Systems (Case 2). For the analysis of the NRTK characteristics, we conducted a long-term experiment with two currently operating NRTK systems of Korea. Two identical GPS receivers were connected to a single antenna on the open sky rooftop. The resulting output, GGA message (fix information) of National Marine Electronics Association (NMEA) sentences, was saved at $1 \mathrm{~Hz}$ rate for 5 days, and the CMR (VRS) and RTCM 2.3 (FKP) were used as a communication protocol to compare different NRTK techniques (see Table 3).

Based on 5 days of NRTK survey in a static mode, the integer ambiguities were resolved to be $99.6 \%$ and $88.6 \%$ on average for VRS and FKP, respectively. The horizontal error of VRS solutions was less than $5 \mathrm{~cm}$ for all epochs with fixed ambiguities, while almost $94 \%$ of FKP solutions were ensured within $5 \mathrm{~cm}$. Therefore, it can be concluded
TABLE 3: Summary of two NRTK surveying [18].

\begin{tabular}{lc}
\hline UTC & 2012-12-15-19 (5 days) \\
\hline Receiver/antenna & Trimble 5700/Leica AT504GG \\
NRTK & VRS/FKP \\
Protocol & CMR (VRS), RTCM2.3 (FKP) \\
GNSS & GPS \\
\hline
\end{tabular}

TABLE 4: Accuracy of NRTK solutions [18].

\begin{tabular}{|c|c|c|c|c|c|}
\hline \multirow[t]{2}{*}{ Component } & \multirow[t]{2}{*}{ Error $(\mathrm{m})$} & \multicolumn{2}{|c|}{$\begin{array}{c}\text { Fixed } \\
\text { solution (\%) }\end{array}$} & \multicolumn{2}{|c|}{$\begin{array}{c}\text { Ambiguity } \\
\text { fixing rate (\%) }\end{array}$} \\
\hline & & VRS & FKP & VRS & FKP \\
\hline North & \multirow{2}{*}{$\leq 0.03$} & 99.7 & 80.8 & \multirow{4}{*}{$99.6 \%$} & \multirow{4}{*}{$88.6 \%$} \\
\hline East & & 100 & 93.1 & & \\
\hline North & \multirow{2}{*}{$\leq 0.05$} & 100 & 93.4 & & \\
\hline East & & 100 & 95.5 & & \\
\hline
\end{tabular}

that VRS is relatively more stable than FKP in a normal condition (see Table 4).

On the other hand, the daily solution shows that ambiguity fixing rate for VRS was consistently stable for the entire period. However, there were some variations in the case of FKP, ranging from $82 \%$ to $91 \%$, particularly almost a $17 \%$ difference on December 17, 2012 (see Figure 4).

To analyze the reason of this gap, we classified the data into two groups, that is, daytime (UTC 0-9, which corresponds to 9 am to $6 \mathrm{pm}$ in local time) and nighttime (UTC 9-24). It shows that the ambiguity fixing rate of FKP during daytime is clearly worse than nighttime, while there is no significant difference for the VRS system (see Table 5). Based on the results, it can be concluded that the FKP system is more sensitive to ionospheric disturbances than VRS. The overall ambiguity fixing rate reached $97.92 \%$ when adding GLONASS in the long run [19]. In addition, FKP shows similar statistics as VRS in positioning accuracy when the integer ambiguities are fixed $(3.7 \pm 2.1 \mathrm{~cm})$. Therefore, GLONASS needs to be included when the FKP system is used, which will certainly increase the stability of the NRTK positioning results. 


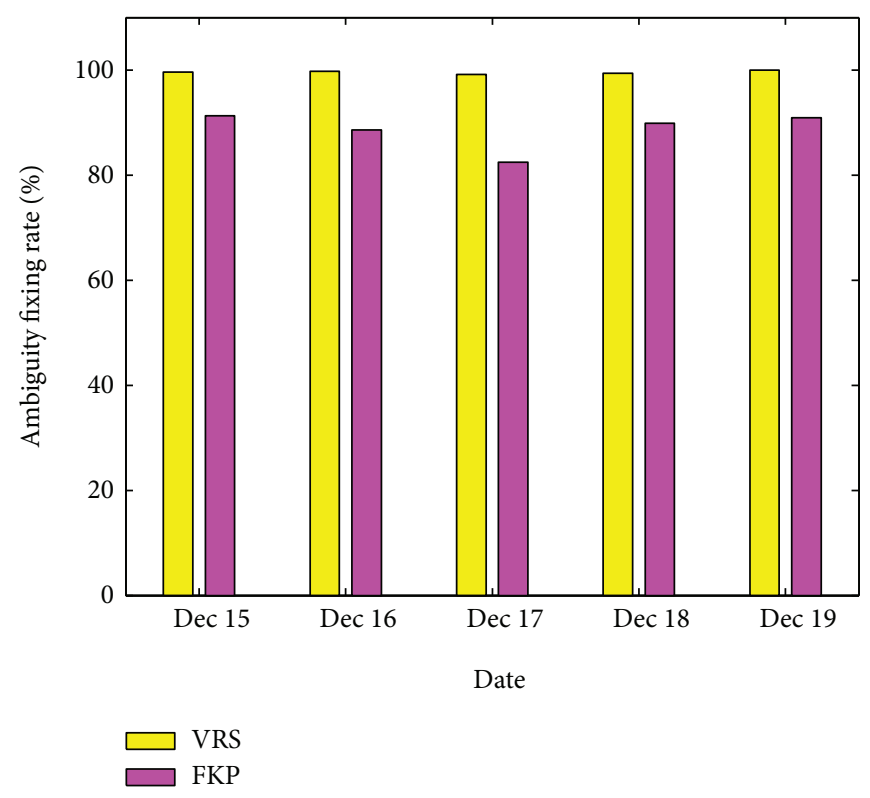

Figure 4: Daily ambiguity fixing rates.

TABle 5: Ambiguity fixing rate by the time of day [18].

\begin{tabular}{llr}
\hline \multirow{2}{*}{ Time } & \multicolumn{2}{l}{ Ambiguity fixing rate (\%) } \\
& VRS & FKP \\
\hline Daytime (UTC 00-09) & 99.2 & 70.9 \\
Nighttime (UTC 09-24) & 99.2 & 94.2 \\
\hline
\end{tabular}

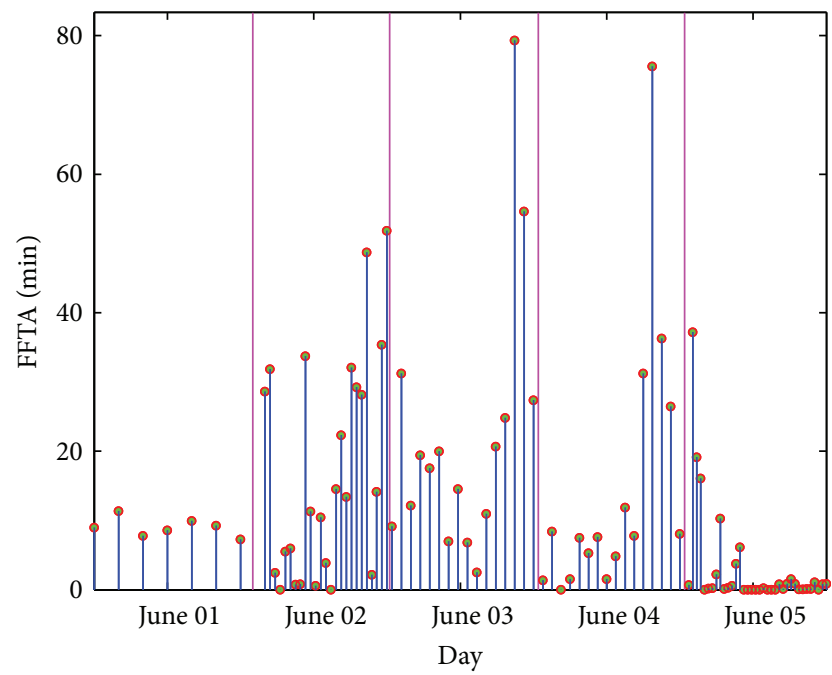

Figure 5: Time to first ambiguity fixing (TTFA) by dates.

3.3. Mobile Application of NRTK (Case 3). The NRTK correction information was applied to kinematic positioning with the same system configuration as the static case. The geodetic class receiver equipped with an internal antenna (Trimble R6) was used at one hand, where NRTK positioning was accomplished within the receiver. As a rover, the low-cost single-frequenccy GNSS receiver (ublox EVK-6T) was connected to the same antenna for checking receiver

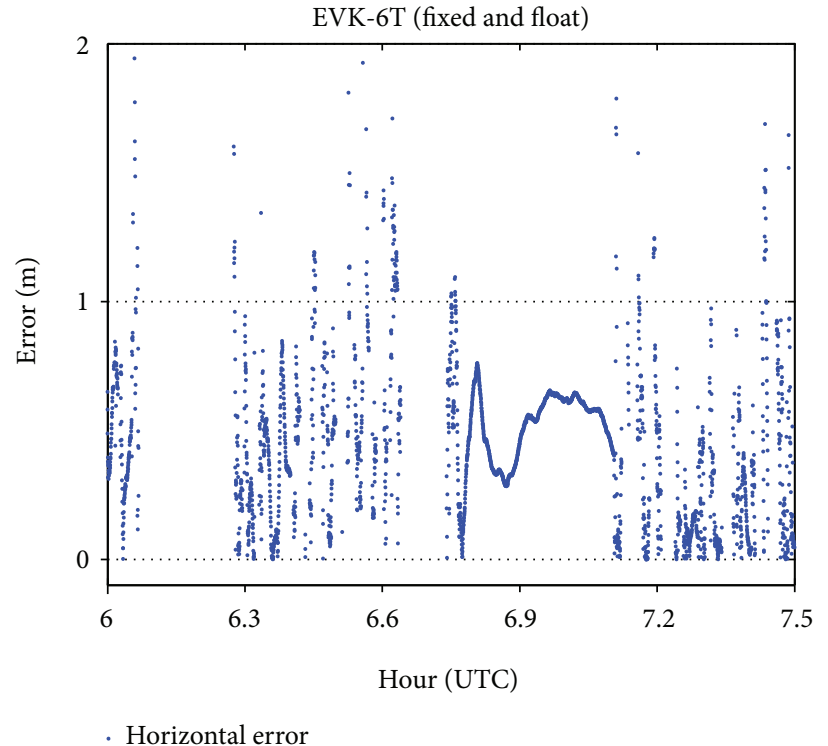

Figure 6: Comparison of NRTK solutions in kinematic mode.

performance, and the NRTK positioning was done by RTKLIB open source software.

Since the ambiguity resolution is the key to NRTK positioning, we analyzed the time to first ambiguity fixing (TTFA) based on 100 times of practices for the low-cost GNSS sensors. The interesting thing is that even the lowcost receiver shows a high performance due to the antenna of GNSS CORS, as was seen in the static case. The results showed unstable duration ranging from several seconds up to 80 minutes, and the integer ambiguities were fixed within several minutes for more than half of epochs. Figure 5 shows the TTFA result that behaves differently depending on the date of observation. The best performance in ambiguity fixing time was accomplished on June 5, 2015. 


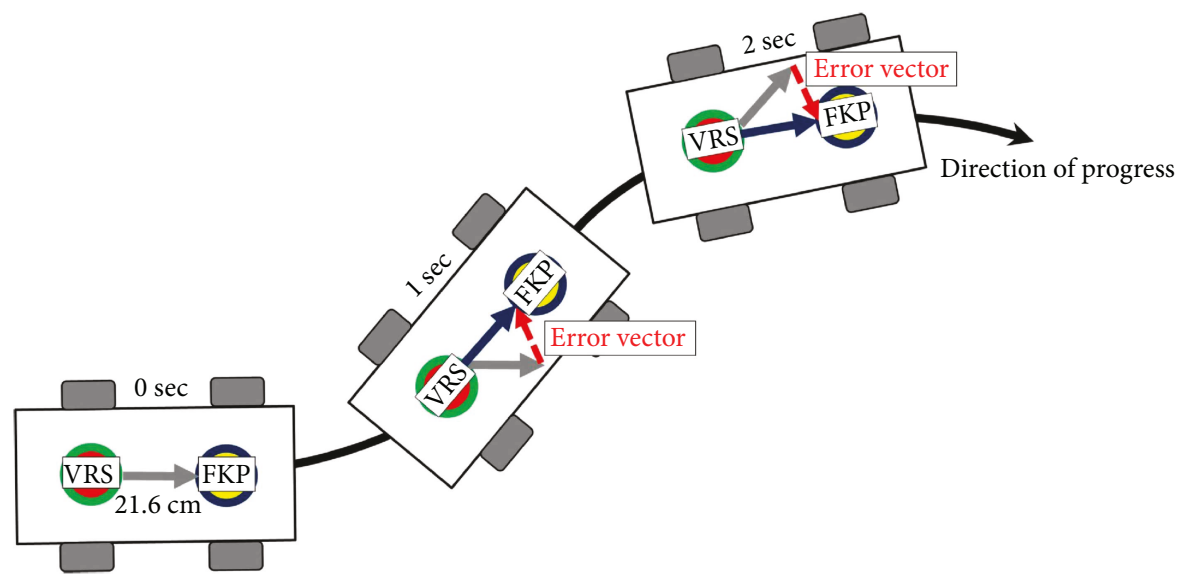

FIGURE 7: System configuration of two NRTK systems.

It is understood that the initialization was not performed properly when reestablishing GNSS receivers to measure TTFA, and the ionospheric condition may be considerably related with the performance of the ambiguity resolution. Therefore, it is concluded that the initial ambiguity fixing can be accomplished within several minutes on average. Since the dual-frequency GNSS receivers usually fix the integer ambiguity in a few seconds, the ambiguity fixing rates can be significantly dropped down for moving singlefrequency receivers.

As an additional experiment, we analyzed the performance of NRTK based on a low-cost GNSS receiver in moving environment but with a patch-type antenna. As mentioned before, the baseline length between detachable GNSS antennas was estimated beforehand. Overall 1043 epochs of measurements were used to estimate the horizontal baseline lengths, resulting in about $1.52 \mathrm{~m}$ with a standard deviation of $0.58 \mathrm{~m}$. Therefore, the bias can affect the NRTK results at a level of about 1 meter. Figure 6 shows the result of the EVK-6T solution with respect to the reference solution by Trimble R6. As expected, it is difficult to resolve the integer ambiguities with low-cost GNS receivers in a moving condition (only $8 \%$ of epochs were fixed to integer). Even the solution with fixed ambiguities shows a similar accuracy as float solution. Since the low-cost receiver can hardly fix the integer ambiguities and may produce outliers in kinematic mode, we set up criteria to eliminate these outliers to calculate the reasonable horizontal baseline lengths. If we assume the outliers to be over $1.5 \mathrm{~m}$, there is no significant difference in the stability between a fixed solution (1.14\%) and a float solution (2.5\%), and the outliers are at a level of a few meters.

Lastly, two geodetic grades of the NRTK receiver with the same type (Trimble R6) were firmly installed on top of a roof of a car with the baseline length of $21.6 \mathrm{~cm}$ (see Figure 7).

Each R6 receiver is connected to a VRS and a FKP server to perform NRTK positioning, and the horizontal baseline vector was estimated to evaluate the performance of both systems. The calibrated baseline length is $21.6 \mathrm{~cm}$ which is considered to be the true value. Assuming the direction
TABLE 6: The combined statistics of NRTK for the horizontal component [3].

\begin{tabular}{lcc}
\hline & Fixed & Float \\
\hline Mean (m) & 0.03 & 2.90 \\
RMSE (m) & 0.07 & 10.36 \\
\hline
\end{tabular}

remains constant during the consecutive epochs, we calculated the horizontal component of the vector for the analysis of NRTK navigation performance. A total of 11 days of NRTK tests were conducted across the country (see the blue lines in Figure 1). Table 6 shows the statistics of relative positioning of two NRTK solutions. The solution can provide a result within several centimeters of precision for a fixed ambiguity, but several meters of uncertainty with about $1.5 \mathrm{~m}$ of bias is expected for the float solution. The integer ambiguity fixing rates are $78.42 \%$ and $68.29 \%$ for VRS and FKP, respectively. When compared with the static NRTK positioning, the ambiguity resolution rates were decreased about 20\%, but still VRS system shows slightly better performance than FKP.

\section{Summary and Conclusions}

We analyzed the effect of the atmospheric disturbances on NRTK positioning as well as the performance of the system in static and/or kinematic mode. A decimeter level accuracy is preferable for the high-accuracy drone navigation for topographic mapping in an open sky. Three cases of experiments were conducted for two currently operating NRTK systems (VRS and FKP) in this study. Since the ambiguity resolution is the most important factor for precision NRTK positioning, we analyzed the performance and the stability of the NRTK system in several aspects: the (1) NRTK techniques, (2) GNSS receiver performance, and (3) status of the rover. In a static mode, there is a difference in the NRTK performance depending on the technique, that is, VRS showed a higher stability than FKP. FKP seems to be influenced by the ionospheric condition when the results are classified into time of observation. 
However, if GLONASS is combined, the stability can be considerably improved as pointed out in the previous section. Even the NRTK solutions by a low-cost receiver do not show an apparent difference in terms of accuracy and stability if connected to a high-quality GNSS antenna. However, the geodetic class antenna is not feasible for the drone navigation; the patch-type antenna was tested as well.

Kinematic NRTK positioning also shows a different characteristic depending on NRTK techniques, and VRS generally performs better than FKP in terms of stability and accuracy. Time of observation does not affect the NRTK performance significantly, but overall the NRTK positioning during daytime shows a better accuracy by several centimeters. The low-cost receiver cannot resolve integer ambiguities in kinematic mode; thus, the performance cannot be guaranteed. Nevertheless, the NRTK positioning of a low-cost receiver provides a submeter level accuracy even under float ambiguities in an open sky, which is a promising result for drone navigation. It should be considered that even a drone navigation sometimes encounters a GNSS signal blockage when modeling buildings in 3-dimensional with detail or taking images under the bridge. Therefore, we need to consider integrating other sensors such as inertial measurement unit (IMU) for the seamless navigation.

The conventional NRTK provides the combined total error of the GNSS observables thus is difficult to model stochastic properties to each specific error source. Another representation of NRTK errors, called the state space representation (SSR), models all physical errors on GNSS observations separately, resulting in effective and flexible correction due to the spatiotemporal knowledge of the parameters [20]. Since a single-frequency operation is possible in SSR, it should be considered for the navigation of drones in the further research.

\section{Data Availability}

The data used to support the findings of this study are available from the corresponding author upon request.

\section{Conflicts of Interest}

The authors declare that there are no conflicts of interest regarding the publication of this paper.

\section{Acknowledgments}

This research was supported by a grant (18RDRP-B07656405) from Regional Development Research Program funded by the Ministry of Land, Infrastructure and Transport of the Korean government.

\section{References}

[1] G. Wubbena, A. Bagge, and M. Schmitz, "Network-based techniques for RTK applications," in Presented at the GPS Symposium, GPS JIN 2001, GPS Society, Japan Institute of Navigation, 2001, pp. 14-16, Tokyo, Japan, 2001.
[2] L. Wanninger, "Introduction to network RTK," in International Association of Geodesy, Working Group 4.5.1: Network RTK, Dresden, Germany, 2008http://www.wasoft.de/e/ iagwg451/wegener/communication.html.

[3] M. H. Kim and T. S. Bae, "Preliminary analysis of networkRTK for navigation," Journal of the Korean Society of Surveying, Geodesy, Photogrammetry and Cartography, vol. 33, no. 5, pp. 343-351, 2015.

[4] B. Park, A Study on Reducing Temporal and Spatial Decorrelation Effect in GNSS Augmentation System [Ph.D. thesis], Seoul National University, Seoul, Korea, 2008.

[5] F. Takac and O. Zelzer, "The relationship between network RTK solutions MAC, VRS, PRS, FKP and i-MAX," in Proceedings of ION GNSS 2008, pp. 348-355, Savannah, GA, USA, 2008.

[6] V. Janssen, "A comparison of the VRS and MAC principles for network RTK," in International Global Navigation Satellite Systems Society, IGNSS Symposium 2009, pp. 1-13, Surfers Paradise, QLD, Australia, 2009.

[7] X. Zou, W. M. Tang, M. R. Ge, J. N. Liu, and H. Cai, "New network RTK based on transparent reference selection in absolute positioning mode," Journal of Surveying Engineering, vol. 139, no. 1, pp. 11-18, 2013.

[8] M. Kim, Algorithm Development of Integrated Navigation System for Ground Vehicle Using Network-RTK [M.S. thesis], Sejong University, Seoul, Korea, 2015.

[9] X. Zou, M. Ge, W. Tang, C. Shi, and J. Liu, "URTK: undifferenced network RTK positioning," GPS Solutions, vol. 17, no. 3, pp. 283-293, 2013.

[10] B. Li, Y. Shen, Y. Feng, W. Gao, and L. Yang, "GNSS ambiguity resolution with controllable failure rate for long baseline network RTK," Journal of Geodesy, vol. 88, no. 2, pp. 99-112, 2014.

[11] T. S. Bae, D. Grejner-Brzezinska, G. Mader, and M. Dennis, "Robust analysis of network-based real-time kinematic for GNSS-derived heights," Sensors, vol. 15, no. 10, pp. 2721527229, 2015.

[12] H. J. Euler and B. E. Zebhauser, "The use of standardized network RTK messages in rover applications for surveying," in Proceedings of the 2003 National Technical Meeting of The Institute of Navigation, vol. 1, pp. 377-384, Anaheim, CA, USA, 2003.

[13] N. Talbot, G. Lu, T. Allison, and U. Vollath, "Broadcast network RTK-transmission standards and results," in Proceedings of the 15th International Technical Meeting of the Satellite Division of The Institute of Navigation (ION GPS 2002), pp. 2379-2387, Portland, OR, USA, 2002.

[14] W. Tang, X. Meng, C. Shi, and J. Liu, "Algorithms for sparse network-based RTK GPS positioning and performance assessment," The Journal of Navigation, vol. 66, no. 3, pp. 335-348, 2013.

[15] C. Wang, Y. Feng, M. Higgins, and B. Cowie, "Assessment of commercial network RTK user positioning performance over long inter-station distances," Journal of Global Positioning Systems, vol. 9, no. 1, pp. 78-89, 2010.

[16] R. Odolinski, “Temporal correlation for network RTK positioning," GPS Solutions, vol. 16, no. 2, pp. 147-155, 2012.

[17] T. S. Bae, "Parametric analysis of the solar radiation pressure model for precision GPS orbit determination," Journal of the Korean Society of Surveying, Geodesy, Photogrammetry and Cartography, vol. 35, no. 1, pp. 55-62, 2017. 
[18] M. H. Kim and T. S. Bae, "Stability assessment of FKP system by NGII using long-term analysis of NTRIP correction signal," Journal of the Korean Society of Surveying, Geodesy, Photogrammetry and Cartography, vol. 31, no. 4, pp. 321-329, 2013.

[19] J. S. Park, Applicability Analysis of FKP for the Cadastral Resurveying and Public Survey through Comparative Analysis of VRS and FKP Position Accuracy [M.S. thesis], University of Seoul, Seoul, Korea, 2014.

[20] G. Wübbena, M. Schmitz, and A. Bagge, "PPP-RTK: precise point positioning using state-space representation in RTK networks," in ION GNSS-05 18th International Technical Meeting, pp. 2584-2594, Long Beach, CA, USA, 2005. 


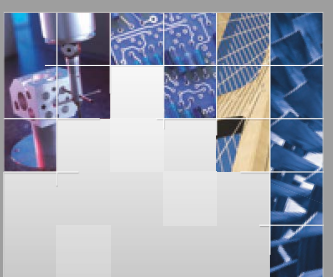

\section{Enfincering}
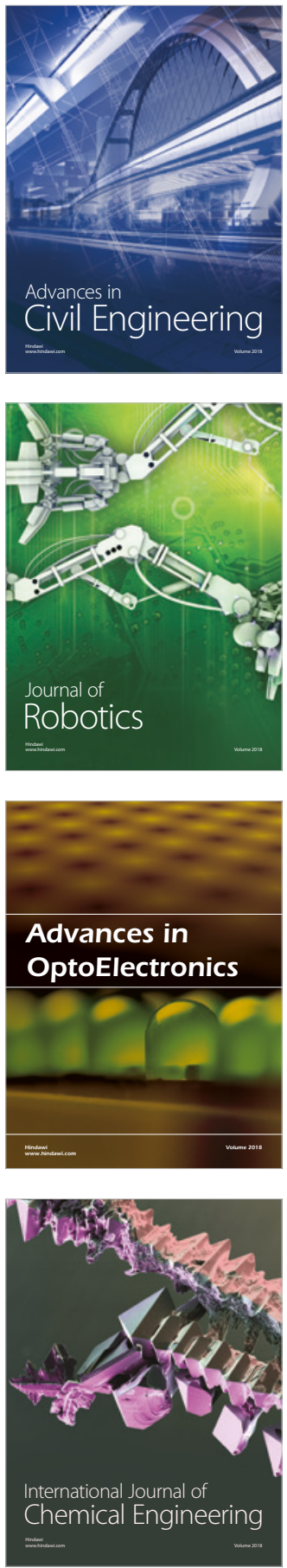

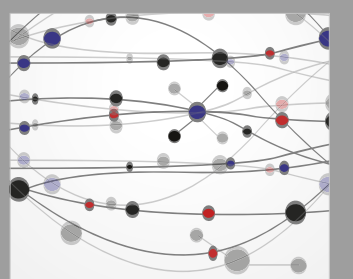

\section{Rotating \\ Machinery}

The Scientific World Journal

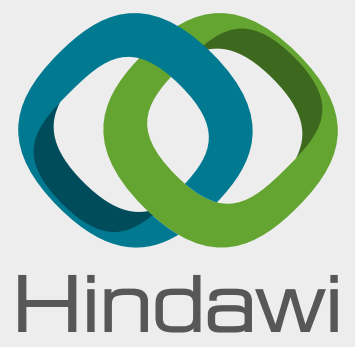

Submit your manuscripts at

www.hindawi.com
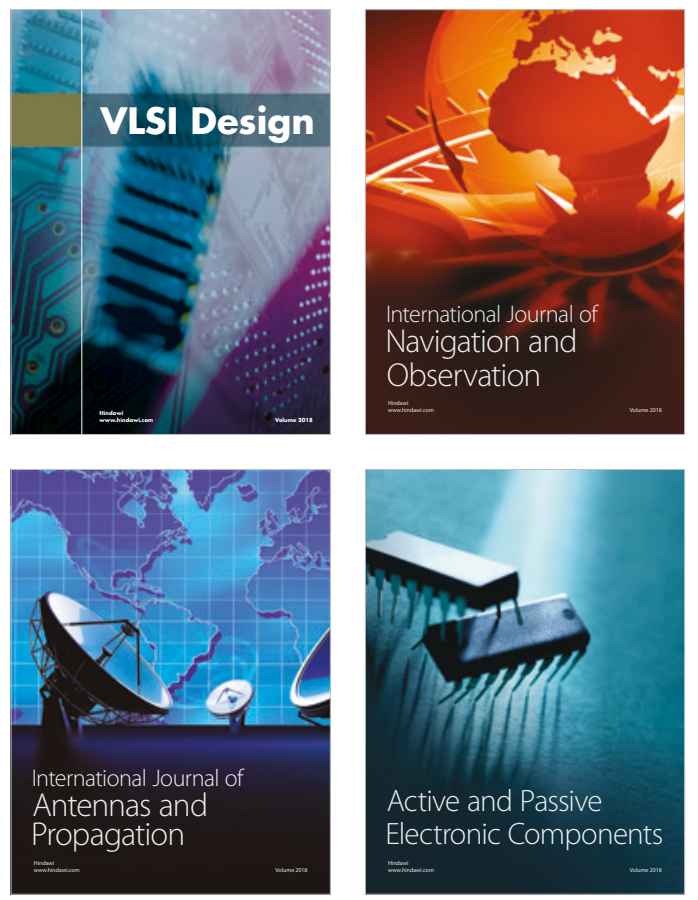
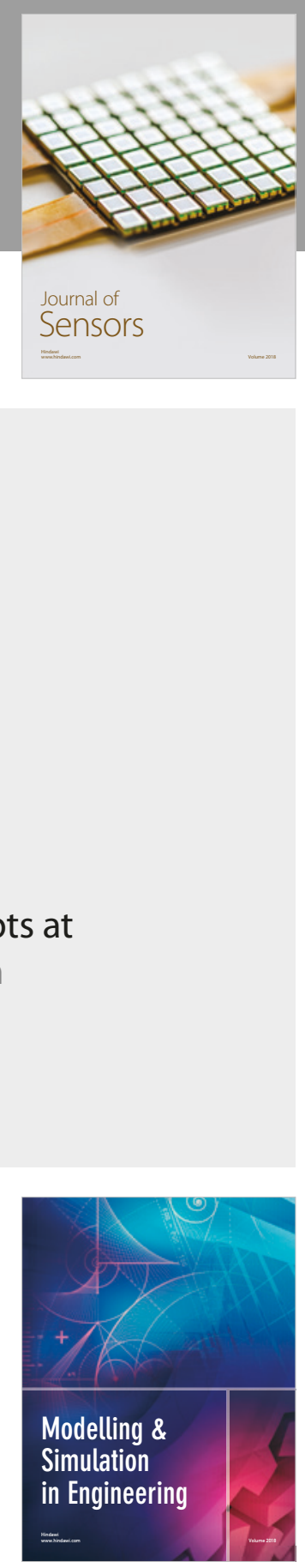

\section{Advances \\ Multimedia}
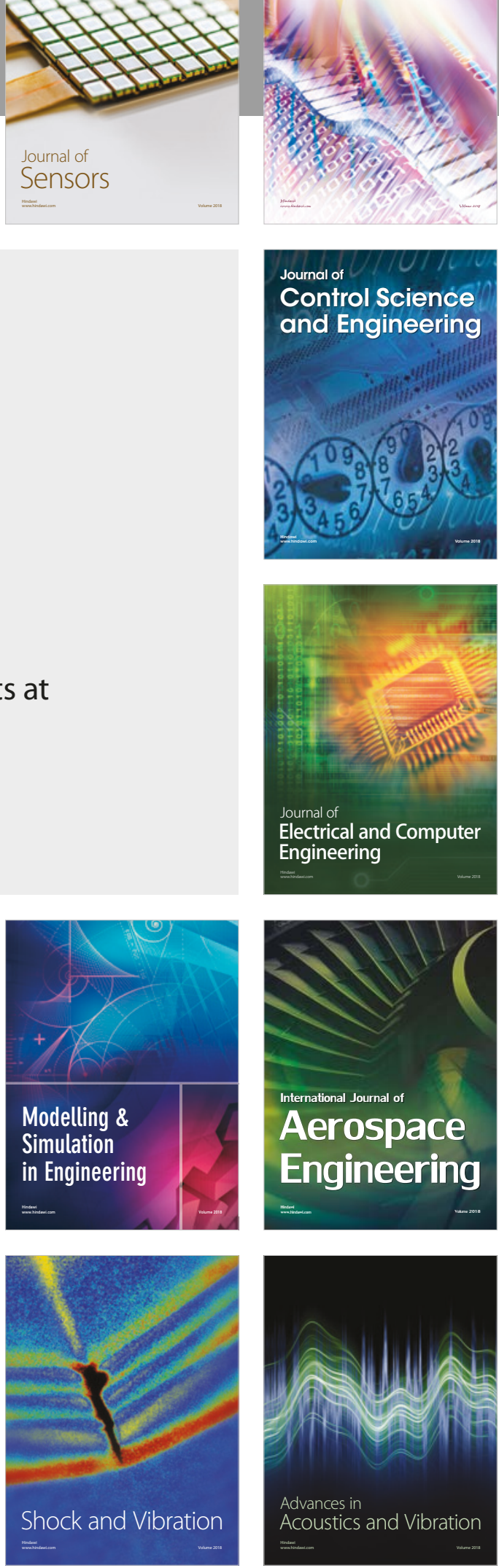